\title{
Building Healthy Workplaces: Time to Act on the Evidence
}

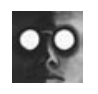

COMMENTARY

\author{
Heather K. Spence Laschinger, RN, $\mathrm{PHD}$ \\ Distinguished University Professor and Associate Director, Nursing Research \\ School of Nursing, Faculty of Health Sciences \\ University of Western Ontario \\ $\propto$
}

\begin{abstract}
Numerous initiatives have been developed to create healthy workplaces in healthcare settings. However, despite these efforts nurses continue to experience negative conditions in their work settings and report challenges to maintaining physical and mental health. Stronger incentives must be put in place to ensure that current healthcare settings meet evidence-based standards for healthy work environments.
\end{abstract}

The AUTHORS OF these two papers provide us with a good overview of healthy workplace issues and describe various initiatives that have been implemented in Canadian healthcare settings in recent years. They focus on two priorities established by the Office of Nursing Policy in Health Canada and championed by Dr. Judith Shamian and Dr. Sandra MacDonald-Rencz - healthy nursing workplaces and effective interdisci- plinary teamwork. Shamian and El-Jardali provide a convincing array of research findings to support the need for these initiatives. It is helpful to see a collation of these various programs in a single article, and it clearly demonstrates that healthy workplaces are on the current policy agenda. The authors outline a number of recommendations for research, policy, practice and education to take this work to the next 
level. In their paper, Clements et al. focus on the importance of teamwork among health providers as a strategy for fostering and sustaining healthy work environments.

It is encouraging to know that these initiatives are under way across the country, but, as the authors point out, it is not clear whether these initiatives have had an impact on direct care providers. Leiter (2006) found that few nurses at the patient care level were familiar with the recommendations of various national reports on the quality of work life in nursing work settings. My own research in the past year has shown that an alarming proportion of nurses (54-66\%) are experiencing severe emotional exhaustion in current hospital settings (Cho et al. 2006; Greco et al. 2006). Three different studies with representative samples of nurse managers, new graduates and nurses in acute care settings revealed that the primary predictor of emotional exhaustion and burnout was excessive workload, followed by a perceived lack of fairness of organizational procedures, poor interpersonal relationships in the work setting, a perceived lack of recognition for their contribution to organizational goals, a lack of congruence between their own and organizational values, and a disempowering work environment. In another 2005 study (Laschinger 2004; Laschinger and Finegan 2005b), fewer than $50 \%$ of nurses surveyed reported that they received the respect they deserved for their contribution to the healthcare in their organization. This result was replicated in the National Survey of Work and Health of Nurses (NSWHN) conducted by Statistics Canada and Canadian Institute for Health Information (CIHI) (2006) in which perceived lack of respect and work overload were significant predictors of nurses' mental and physical health.
Clearly, we have a long way to go in creating healthy work environments in nursing as these results show that basic human factors that foster individual health and well-being are still lacking in current nursing work environments. It is important to pay attention to these basic psychosocial aspects of healthy work environments as well as the physical health aspects of nursing work settings. In all of the above-mentioned studies, the extent to which nurses felt they had access to empowering work structures, such as information, support, resources and opportunities to learn and grow, was strongly predictive of nurses' feelings of being respected in their workplace, their burnout levels and their perceived fit with their work environment. Creating empowering work environments is the mandate of management. It must be supported at higher levels of the organization and monitored to ensure that these conditions are in place. While empowerment is only one of the many important components of a healthy work environment, it has been shown to be fundamental to nurses' health and wellbeing and an important determinant of job satisfaction, organizational commitment and turnover (Nedd 2006).

We know that the nursing profession is currently experiencing a severe nursing shortage, with many nurses approaching retirement and fewer people entering the profession. Many are leaving the profession altogether. Furthermore, Boychuk Duchscher (2001) and a Canadian Nurses Association report (2000) showed that many new graduates are leaving their jobs within two years of graduation. All these factors will intensify the nursing shortage and add to the stressful nature of nursing working conditions. Burnout, the inevitable result of long-term exposure to stressful working conditions, is a precursor of job dissatisfac- 
tion and turnover, something we can ill afford with the current nursing shortage. We have considerable evidence and theory that articulate factors in the workplace that contribute to this syndrome; this knowledge can guide efforts to change things for the better. We also know that burnout has negative health effects for both nurses and the patients they serve (Laschinger and Finegan 2005a; Leiter et al. 1998). Therefore, workplace initiatives that address this issue are urgently needed if we are to sustain a healthy nursing workforce that will ensure that patients will continue to receive the high-quality care they deserve.

Shamian and El-Jardali note a need for the evaluation of current healthy workplace initiatives and for employers to be made accountable for ensuring that their organizations meet standards for healthy workplaces. Since 1999, Canadian Council on Health Services Accreditation (CCHSA) standards have included work-life quality indicators that increase the likelihood that organizations will pay more attention to these issues. However, since the accreditation process is voluntary and funding and approval are not tied to meeting these criteria, it is difficult to ensure that these conditions are met consistently across healthcare settings. There is a need for a mechanism that requires organizations to demonstrate that these standards are in place and that they are effective in promoting employee health.

The Quality Worklife-Quality Healthcare Collaborative (QWQHC) is a promising initiative that brings together a coalition of 11 national health partners to develop a pan-Canadian strategy for translating evidence-based approaches to building and sustaining healthy work environments into practice at the direct care level. An important component of this initiative will be to put in place a mechanism for moni- toring the quality of work life across the country over time, using a common measure or set of indicators. This will provide a basis for monitoring the effects of healthy work environment programs over time using a common metric and provide direction for any necessary improvements that may be required. This approach will permit national comparisons of work-life quality and could serve as a national report card on healthy work environments in Canada. This will be a major improvement on current practice, where there is little consistency in measures across settings - making it difficult for organizations to benchmark their progress in this area. This common measure could even be used by all healthcare organizations on an annual basis as part of their quality-improvement programs to enable them to track their own progress and to compare their results with those of similar organizations across the country. Ultimately, these results could be collected in a national database and used in research to study the impact of these work-life conditions on provider, client and system outcomes.

Ideally, such a measure is based on an evidence-based explicit theoretical framework that articulates the relevant components of a healthy workplace and their interrelationships. The Pulse measure to be used by the QWQHC is grounded in the CCHSA healthy workplace framework. The national nurses' health survey conducted by Statistics Canada and CIHI is another source of data on the health effects of worklife interventions that could be used over time to monitor nurses' health. Indeed, this survey could be extended to include all health providers in the system, which would provide a comprehensive assessment of working conditions in our healthcare sector. The QWQHC is the first national initiative involving a powerful mix of stakeholders at a 
variety of levels committed to putting knowledge into action. It will be crucial to ensure that this effort receives sustainable funding to enable them to continue their work.

Clements et al. describe the importance of effective teamwork in ensuring high-quality work life and positive patient outcomes. Interdisciplinary silos and disciplinary turf wars have contributed to workplace stress and affected patient care quality in the past, and efforts are being made to promote effective interdisciplinary education and practice. These efforts are strongly supported by policy groups, such as Health Canada, that have launched a number of initiatives intended to improve teamwork among the health professions. Evidence to support this work was established in the NSWHN study where poor nurse/physician collaboration was found to be a significant predictor of nurses' mental and physical health (Statistics Canada and CIHI 2006).

Interestingly, the proposed solutions for improving teamwork mirror those for ensuring healthy work environments are in place. This is logical since effective collaboration in teams is an important component of healthy workplaces. There is evidence that effective collaboration among health professionals has positive effects on provider, client and system outcomes. However, it is important that all team members retain their professional identity and are clear about what they bring to the healthcare process. Effective interdisciplinary collaboration requires mutual respect for all team members' skills and expertise and a willingness to listen to other points of view in the process of planning and providing optimal patient care. The authors suggest several strategies that will lay the foundation for mutually respectful, effective healthcare teams. These relationships must be supported and, indeed, demanded in all healthcare settings if patients are to benefit from the expertise of all health professionals and continue to receive highquality patient care.

\section{References}

Boychuk Duchscher, J.E. 2001. "Out in the real world. Newly Graduate Nurses in Acute-Care Speak Out." Journal of Nursing Administration 31(9): 426-439.

Canadian Nurses Association. 2000. Labour Market Integration of Graduates in Nursing in Canada 19861997. A report prepared by the Canadian Council on Social Development for the Canadian Nurses Association. Ottawa, ON: Author.

Cho, J., H.K.S. Laschinger and C. Wong. 2006. "Workplace Empowerment, Work Engagement and Organizational Commitment of New Graduate Nurses." Canadian Journal of Nursing Leadership 19(3): 43-60.

Greco, P., H.K.S. Laschinger and C. Wong. 2006. "Leader Empowering Behaviours, Staff Nurse Empowerment, and Work Engagement/Burnout." Canadian Journal of Nursing Leadership 19(4): 42-57.

Laschinger, H.K.S. 2004. “Hospital Nurses' Perceptions of Respect and Organizational Justice." Journal of Nursing Administration 34: 354-64.

Laschinger, H.K.S. and J. Finegan. 2005a. "Empowering Nurses for Work Engagement and Health in Hospital Settings." Journal of Nursing Administration 35: 439-49.

Laschinger, H.K.S. and J. Finegan. 2005b. "Using Empowerment to Build Trust and Respect in the Workplace: A Strategy for Addressing the Nursing Shortage." Nursing Economics 23: 6-13.

Leiter, M.P. 2006. Nursing Environments: Knowledge to Action. Ottawa: Health Policy Research Program, Health Canada.

Leiter, M.P., P. Harvie and C. Frizzell. 1998. "The Correspondence of Patient Satisfaction and Nurse Burnout." Social Science and Medicine 47: 1611-17.

Nedd, N. 2006. "Perceptions of Empowerment and Intent to Stay." Nursing Economics 24(1): 13-18.

Statistics Canada and Canadian Institute for Health Information. 2006. A Summary of Highlights from the 2005 National Survey of the Work and Health of Nurses. Ottawa: Author. 